\title{
Prognostic significance of p16 \& p53 immunohistochemical expression in triple negative breast cancer
}

\author{
Atif Ali Hashmi', Samreen Naz', Shumaila Kanwal Hashmi', Zubaida Fida Hussain ', Muhammad Irfan', \\ Erum Yousuf Khan ${ }^{1}$, Naveen Faridi ${ }^{1}$, Amir Khan $^{3^{*}}$ (D) and Muhammad Muzzammil Edhi ${ }^{4}$
}

\begin{abstract}
Background: 16 and p53 genes are frequently mutated in triple negative breast cancer \& prognostic value of these mutations have been shown; however, their role as immunohistochemical overexpression has not been fully validated. Therefore we aimed to evaluate the association of p16 and p53 overexpression in triple negative breast cancer with various prognostic parameters.

Methods: Total 150 cases of triple negative breast cancers were selected from records of pathology department archives that underwent surgeries at Liaquat National hospital, Karachi from January 2008 till December 2013. ER, PR and Her2neu immunohistochemistry were re-performed to confirm triple negative status. p16 \& p53

immunohistochemistry was performed on all cases and association with various clinicopathologic parameters was determined.

Results: Mean age of the patients involved in the study was 48.9 years. Most of the patients presented at stage T2 with a high mean ki67 index i.e. $46.9 \% .42 .7 \%$ of cases had nodal metastasis. Although $84 \%$ cases were of invasive ductal carcinoma; however a significant proportion of cases were of metaplastic histology (9.3\%). Fifty-one percent (76 cases) of cases showed positive p53 expression while 49\% (74 cases) were negative. Higher percentage of p53 expression was found to correlate with higher T stage, high ki67 index and higher nodal stage. On the other hand, strong intensity of p53 expression was positively correlated with higher tumor grade and ki67 index. Seventy-one percent (98 cases) of cases showed positive p16 expression, whereas 24.8\% (34 cases) were negative and 3.6\% (5 cases) showed focal positive p16 expression. However, no significant association was found between p16 expression and various clinical and pathologic parameters. Similarly, no significant association of either p16 or p53 over-expression was noted with recurrence status of patients.
\end{abstract}

Conclusion: On the basis of significant association of p53 over-expression with worse prognostic factors in triple negative breast cancer, therefore we suggest that more large scale studies are needed to validate this finding in loco-regional population. Moreover, high expression of p16 in triple negative breast cancer suggests a potential role of this biomarker in triple negative breast cancer pathogenesis which should be investigated with molecular based research in our population.

Keywords: p53, p16, Triple negative breast cancer

\footnotetext{
* Correspondence: dramirkhan04@gmail.com

${ }^{3}$ Kandahar University, North, Kandahar 3802, Afghanistan

Full list of author information is available at the end of the article
}

(c) The Author(s). 2018 Open Access This article is distributed under the terms of the Creative Commons Attribution 4.0 International License (http://creativecommons.org/licenses/by/4.0/), which permits unrestricted use, distribution, and reproduction in any medium, provided you give appropriate credit to the original author(s) and the source, provide a link to the Creative Commons license, and indicate if changes were made. The Creative Commons Public Domain Dedication waiver (http://creativecommons.org/publicdomain/zero/1.0/) applies to the data made available in this article, unless otherwise stated. 


\section{Background}

Triple negative breast cancers (TNBC) comprise approximately $20 \%$ of breast cancers worldwide while a higher frequency of TNBC were noted in south Asian population [1, 2]. American Society of Clinical Oncology (ASCO)/ College of American Pathologists (CAP) defines TNBC as those breast cancers which shows $<1 \%$ estrogen receptor (ER)/ progesterone receptor (PR) expression by immunohistochemistry (IHC) and either $0-1+$ Her2neu by IHC or $2+$ with negative fluorescent insitu hybridization (FISH) [3-5]. TNBC are typically high grade and associated with worse prognostic and predictive factors and are therefore focus of current clinical research [6, 7]. Moreover TNBC are not a single clinical entity and various subtypes of TNBC have been defined based on molecular studies including basal like subtypes, immunomodulatory, mesenchymal, mesenchymal stem-like, luminal androgen subtypes, claudin low and interferon rich subtypes $[8,9]$. Basal like subtype of TNBC is a molecularly defined subtype of TNBC with high expression of basal cytokeratins (CK5/6) and epidermal growth factor receptor (EGFR) and it correlates with IHC expression of CK5/6, [10, 11].

p16 and p53 are proteins which are involved in two major cell cycle control pathways frequently targeted in human tumorigenesis. Virtually all human cancers show dysregulation of either p16 or p53 pathways [12-14]. Prognostic value of p16 and p53 mutations in breast cancer has been shown in various studies $[15,16]$ however their role as IHC overexpression in TNBC has not been fully understood. Therefore, we aimed to evaluate the association of p16 and p53 overexpression in TNBC with various prognostic parameters like tumor stage, tumor grade, nodal metastasis and lymphovascular invasion.

\section{Methods}

The study included 150 cases of TNBC that had their primary resection at Liaquat National hospital from January 2008 till December 2013 over duration of 6 years. Type of surgeries included wide local excisions and simple mastectomies with sentinel lymph node dissection or wide local excision with axillary dissection and modified radical mastectomies. The approval of the study was taken from institutional research and ethical review committee. At the time of surgery, an informed written consent was taken from each patient. Clinical records of all patients were evaluated and histopathological findings like tumor type, grade and stage were recorded after reviewing $\mathrm{H}$ \& E slides. Moreover, representative sections of all tumors were re-cut for $\mathrm{H} \& \mathrm{E}$ and IHC staining. ER, PR, Her2neu, Ki67, CK5/6, p16 and p53 IHC were performed on representative sections.
ER, PR, Her2neu and Ki67 IHC were performed using DAKO antibodies as under, with EnVision ${ }^{\text {тM }}$ FLEX, high pH DAKO kit according to manufacturer's protocol.

1. FLEX Monoclonal Rabbit Anti-human Estrogen Receptor alpha, Clone EP1.

2. FLEX Monoclonal Mouse Anti-human Progesterone receptor clone PgR 636

3. Polyclonal Rabbit Anti-human c-erbB-2 oncoprotein

4. FLEX Monoclonal mouse Anti-human Ki67 Antigen clone MIB-1

For ER and PR IHC, nuclear staining in more than $1 \%$ cancer cells was taken as positive expression [4]. For, her2neu IHC, staining was scored as per CAP guidelines into 1 + (weak), 2+ (intermediate) and 3+ (strong) expression. Cases with intermediate $(2+)$ expression were subjected to Fluorescent insitu hybridization (FISH) testing and results were reported as amplified or non-amplified as per CAP guidelines [5].

Ki67 IHC was interpreted on the basis of average percentage of positively stained cancer cells. Only nuclear expression was taken as positive. At-least 1000 cancer cells were counted in five different areas of tumor and average percentage of positively stained cancer cells were recorded and then categorized.

CK5/6 IHC was performed by using FLEX Monoclonal Mouse Anti-human Cytokeratin 5/6, clone D5/16 B4 by DAKO envision method according to manufacturers protocol. Moderate to strong cytoplasmic and membranous staining in more than $10 \%$ cells was taken as positive expression. Tumors with positive CK5/6 were labeled as basal phenotype and those with negative CK5/6 expression were called as non-basal phenotype.

p53 IHC was performed using DAKO EnVision method using DAKO anti-human p53 protein, clone DO-7 according to manufacturers protocol. Nuclear staining for p53 was both quantitatively and qualitatively evaluated. Intensity of staining was categorized into no staining (0), weak $(1+)$, intermediate $(2+)$, strong $(3+)$ while percentage of positively stained cells were measured as continuous variable. Intermediate to strong staining in $>10 \%$ cancer cells was considered positive while no staining or weak staining in $<10 \%$ cancer cells was taken as negative (Fig. 1). Moreover, p53 immunostaining was also categorized according to percentage of staining cells into different groups.

p16 antibody was purchased from Roche Ventana and IHC was performed using antibody CINtec $\mathrm{R}$ p $16^{\mathrm{INK} 4 \mathrm{a}}$, clone $\mathrm{E} \mathrm{H}_{4}^{\mathrm{Tm}}$ according to manufacturers protocol. Tonsils and carcinoma cervix was taken as positive controls. Both nuclear and cytoplasmic staining was evaluated. Intensity of staining was categorized into no staining (0), 


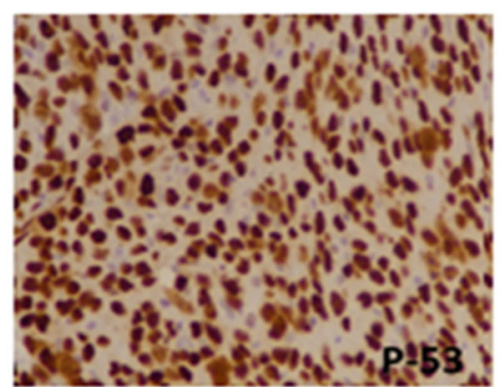

Positive 40x

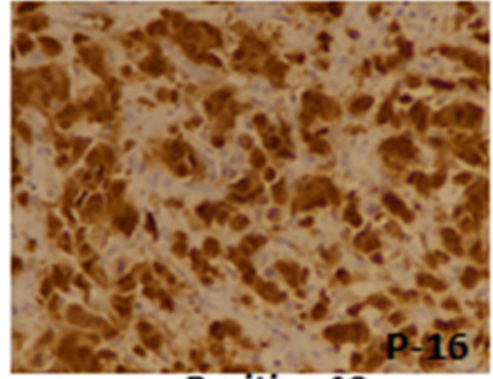

Positive 40x

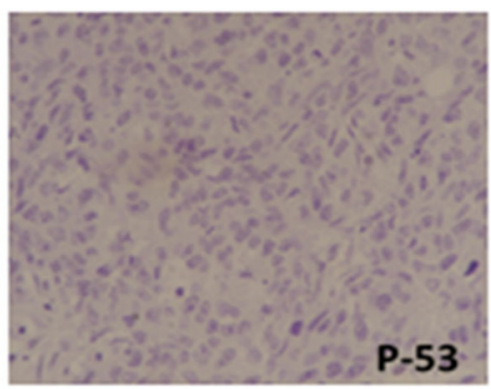

Negative 40x

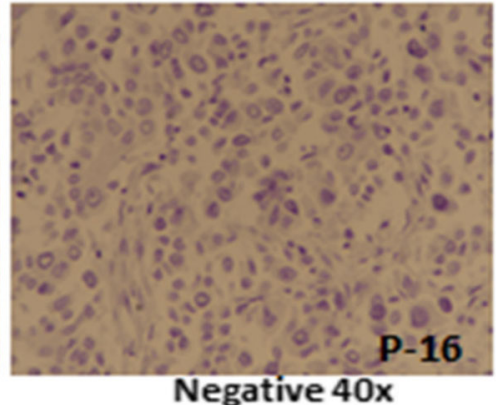

Fig. 1 p53 \& p16 expression in triple negative breast cancer

weak $(1+)$, intermediate $(2+)$, strong $(3+)$ while percentage of positively stained cells were measured as continuous variable. Intermediate to strong staining in $>10 \%$ cancer cells was considered positive while weak to intermediate staining in $<10 \%$ cancer cells was taken as focal positive (Fig. 1). Similarly, p16 immunostaining was also categorized according to percentage of staining cells into different groups.

Patient's clinical records were reviewed to evaluated recurrence and survival status. Time from surgery till death due to disease, local recurrence, distant metastasis or last follow was defined as disease free survival.

Statistical package for social sciences (SPSS 21) was used for data entry and analysis. We calculated mean and standard deviation for quantitative variables while, frequency and percentage were evaluated for qualitative variables. Chi-square was applied to determine association between the variables. Student $t$ test or Mann witney test were applied to compare difference in means among groups where necessary. $P$-value of $\leq 0.05$ was taken as significant. Survival curves were plotted using Kaplan- Meier method and the significance of difference between survival curves were evaluated using log-rank ratio.

A sample size of 150 achieves $79 \%$ power to detect an effect size (W) of 0.2994 using a 6 degrees of freedom Chi-Square Test with a significance level (alpha) of 0.05000 .

\section{Results}

Mean age of the patients involved in the study was 48.9 years and most common age group was $31-50$ years. Most of the patients presented at stage T2 with a high mean ki67 index i.e. $46.9 \%$. $42.7 \%$ of cases had nodal metastasis. Although $84 \%$ cases were of conventional invasive ductal carcinoma, NST; however a significant proportion of cases were of metaplastic histology (9.3\%). Majority cases were of high grade (86.7\% grade III). Most tumors show lymphocytic infiltration and necrosis. Most of the tumors lack insitu component (61\%) and only $10 \%$ cases were of basal phenotype (CK5/6 positive). Local recurrence or late distant metastasis was noted in $17.8 \%$ of cases (Table 1).

Fifty-one percent (76 cases) of TNBC showed positive p53 expression while $49 \%$ (74 cases) were negative. Further categorization on the basis of percentage of p53 expression revealed; $36 \%$ (54 cases) showed high p53 expression (> 70\%), 12\% (18 cases) revealed $51-70 \%$ p53 expression, $12 \%$ (18 cases) showed 11-50\% p53 expression and $40 \%$ (60 cases) showed either no p53 expression or weak expression in less than 10\% tumor cells. 30.7\% (46 cases) showed no p53 expression while 14\% (21 cases), $17.3 \%$ (26 cases) and 38\% (57 cases) revealed weak, intermediate and strong 53 expression respectively. Correlation of percentage of $\mathrm{p} 53$ expression with various clinicopathologic variables revealed significant associations (Table 2). High p53 expression was found to correlate with higher T stage, high ki67 index and higher nodal stage. Although 
Table 1 Clinicopathologic characteristics of triple negative breast cancer

\begin{tabular}{|c|c|}
\hline & $\mathrm{n}(\%)$ \\
\hline$\overline{\text { Age }(\text { years) }}{ }^{\circ}$ & $48.85 \pm 11.49$ \\
\hline \multicolumn{2}{|l|}{ Age groups } \\
\hline$\leq 30$ years & $5(3.3)$ \\
\hline $31-50$ years & $84(56)$ \\
\hline$>50$ years & $61(40.7)$ \\
\hline Tumor size(Unit) $^{\circ}$ & $4.01 \pm 1.99$ \\
\hline \multicolumn{2}{|c|}{ Tumor stage/tumor size } \\
\hline T1 & $7(4.7)$ \\
\hline $\mathrm{T} 2$ & $116(77.3)$ \\
\hline $\mathrm{T} 3 / \mathrm{T} 4$ & $27(18)$ \\
\hline Ki67 Index (\%) & $46.89 \pm 23.88$ \\
\hline \multicolumn{2}{|l|}{ ki67 index groups } \\
\hline$\leq 15 \%$ & $17(11.3)$ \\
\hline $16-24 \%$ & $8(5.3)$ \\
\hline $25-44 \%$ & $45(30)$ \\
\hline$>44 \%$ & $80(53.3)$ \\
\hline \multicolumn{2}{|l|}{ Nodal Status } \\
\hline Positive & $64(42.7)$ \\
\hline Negative & $86(57.3)$ \\
\hline \multicolumn{2}{|l|}{ Nodal Stage } \\
\hline No & $88(58.7)$ \\
\hline N1 & $30(20)$ \\
\hline N2 & $13(8.7)$ \\
\hline N3 & $19(12.7)$ \\
\hline \multicolumn{2}{|l|}{ Histological Subtypes } \\
\hline IDC & $127(84.7)$ \\
\hline Papillary & $6(4)$ \\
\hline Medullary & $1(0.7)$ \\
\hline Metaplastic & $14(9.3)$ \\
\hline Mixed & $2(1.3)$ \\
\hline \multicolumn{2}{|l|}{ Tumor Grade } \\
\hline Grade-I & $1(0.7)$ \\
\hline Grade-II & $19(12.7)$ \\
\hline Grade-III & $130(86.7)$ \\
\hline \multicolumn{2}{|c|}{ Lymphocytic infiltration } \\
\hline Absent & $15(10)$ \\
\hline Moderate & $110(73.3)$ \\
\hline Severe & $25(16.7)$ \\
\hline \multicolumn{2}{|c|}{ Lymhovascular Invasion } \\
\hline Present & $36(24)$ \\
\hline Absent & $114(76)$ \\
\hline \multicolumn{2}{|c|}{ Dermal Lymphatic invasion } \\
\hline Present & $10(6.7)$ \\
\hline Absent & $140(93.3)$ \\
\hline
\end{tabular}


Table 1 Clinicopathologic characteristics of triple negative breast cancer (Continued)

\begin{tabular}{|c|c|}
\hline & n (\%) \\
\hline \multicolumn{2}{|l|}{ Type of Surgery } \\
\hline Modified radical mastectomy & $94(62.7)$ \\
\hline Simple mastectomy with sentinel lymph node dissection & $42(28)$ \\
\hline Wide local excision & $14(9.3)$ \\
\hline \multicolumn{2}{|l|}{ Necrosis } \\
\hline Absent & $21(14)$ \\
\hline Moderate & $90(60)$ \\
\hline Severe & $39(26)$ \\
\hline \multicolumn{2}{|l|}{ Fibrosis } \\
\hline Mild & $42(28)$ \\
\hline Moderate & $88(58.7)$ \\
\hline Severe & 20(13.3) \\
\hline \multicolumn{2}{|l|}{ Insitu component } \\
\hline Present & $58(38.7)$ \\
\hline Absent & $92(61.3)$ \\
\hline \multicolumn{2}{|l|}{ Pagetoid Spread } \\
\hline Present & $2(1.3)$ \\
\hline Absent & 148(98.7) \\
\hline \multicolumn{2}{|l|}{ Perinodal extension } \\
\hline Present & $30(20)$ \\
\hline Absent & $120(80)$ \\
\hline \multicolumn{2}{|l|}{ Triple negative phenotype } \\
\hline Basal & 16(10.7) \\
\hline Non-basal & 134(89.3) \\
\hline \multicolumn{2}{|l|}{ Adjuvant chemotherapy $(n=101)$} \\
\hline Yes & $98(97)$ \\
\hline No & $3(3)$ \\
\hline \multicolumn{2}{|l|}{ Adjuvant radiation $(n=101)$} \\
\hline Yes & $69(68.3)$ \\
\hline No & $32(31.7)$ \\
\hline \multicolumn{2}{|l|}{ Recurrence $(n=101)$} \\
\hline Yes & 18(17.8) \\
\hline No & $83(82.2)$ \\
\hline
\end{tabular}

Mean \pm SD

not statistically significant, but higher p53 expression was also noted in medullary and metaplastic cancers ( $p$-value 0.06). On the other hand, intensity of p53 expression was positively correlated with tumor grade and ki67 index; however, correlation with other parameters was not significant (Table 3).

Seventy-one percent (98 cases) of TNBC showed positive p16 expression, whereas $24.8 \%$ (34 cases) were negative and $3.6 \%$ (5 cases) showed focal positive p16 expression. $24.8 \%$ (34 cases) revealed no p16 expression while $10.9 \%$ (15 cases), $28.5 \%$ (39 cases) and $35.8 \%$ ( 49 cases) showed weak, intermediate and strong p16 expression respectively. 28.5\% (39 cases) revealed no expression or weak expression in $<10 \%$ cancer cells, $15.3 \%$ (21 cases) showed $11-50 \%$ expression, $13.1 \%$ (18 cases) showed $51-70 \%$ expression while $43.1 \%$ (59 cases) revealed $>70 \%$ p16 expression. However, no significant association was found between p16 expression and various clinical and pathologic parameters (Table 3). Similarly, no significant 
Table 2 Association of percentage of p53 overexpression with various clinical \& pathological parameters

\begin{tabular}{|c|c|c|c|c|c|c|}
\hline \multirow{3}{*}{ Age groups } & \multicolumn{5}{|l|}{$\mathrm{n}(\%)$} & \multirow{2}{*}{$\begin{array}{l}P \text { - } \\
\text { Value }\end{array}$} \\
\hline & $\leq 10 \%(n=60)$ & $11-50 \%(n=18)$ & $51-70 \%(n=18)$ & $>70 \%(n=54)$ & Total $(n=150)$ & \\
\hline & & & & & & \\
\hline & $2(3.3)$ & $0(0)$ & $0(0)$ & $3(5.6)$ & $5(3.3)$ & 0.217 \\
\hline $31-50$ years & $34(56.7)$ & $7(38.9)$ & $8(44.4)$ & $35(64.8)$ & $84(56)$ & \\
\hline$>50$ years & $24(40)$ & $11(61.1)$ & 10(55.6) & 16(29.6) & $61(40.7)$ & \\
\hline \multicolumn{7}{|c|}{ Tumor stage/tumor size } \\
\hline $\mathrm{T} 1(\leq 2 \mathrm{~cm})$ & $3(5)$ & $6(33.3)$ & $3(16.7)$ & 14(25.9) & $26(17.3)$ & 0.020 \\
\hline $\mathrm{T} 2(2.1-5.0 \mathrm{~cm})$ & $36(60)$ & $6(33.3)$ & 10(55.6) & $27(50)$ & 79(52.7) & \\
\hline $\mathrm{T} 3(>5.0 \mathrm{~cm})$ & $21(35)$ & $6(33.3)$ & $5(27.8)$ & $13(24.1)$ & $45(30)$ & \\
\hline \multicolumn{7}{|l|}{ ki67 index groups } \\
\hline$\leq 15 \%$ & $6(10)$ & $6(33.3)$ & $4(22.2)$ & $1(1.9)$ & 17(11.3) & 0.000 \\
\hline $16-24 \%$ & $2(3.3)$ & $2(11.1)$ & $3(16.7)$ & $1(1.9)$ & $8(5.3)$ & \\
\hline $25-44 \%$ & 19(31.7) & $6(33.3)$ & $7(38.9)$ & 13(24.1) & $45(30)$ & \\
\hline$>44 \%$ & $33(55)$ & $4(22.2)$ & $4(22.2)$ & $39(72.2)$ & $80(53.3)$ & \\
\hline \multicolumn{7}{|l|}{ Nodal Status } \\
\hline Positive & $30(50)$ & $5(27.8)$ & 10(55.6) & 19(35.2) & $64(42.7)$ & 0.144 \\
\hline Negative & $30(50)$ & 13(72.2) & $8(44.4)$ & $35(64.8)$ & $86(57.3)$ & \\
\hline \multicolumn{7}{|l|}{ Nodal Stage } \\
\hline No & $32(53.3)$ & 13(72.2) & $8(44.4)$ & $35(64.8)$ & 88(58.7) & 0.022 \\
\hline N1 & $15(25)$ & $3(16.7)$ & $2(11.1)$ & 10(18.5) & $30(20)$ & \\
\hline N2 & $3(5)$ & 1(5.6) & $7(38.9)$ & $2(3.7)$ & $13(8.7)$ & \\
\hline N3 & 10(16.7) & $1(5.6)$ & $1(5.6)$ & $7(13)$ & 19(12.7) & \\
\hline \multicolumn{7}{|c|}{ Histological Subtypes } \\
\hline IDC & $51(85)$ & 14(77.8) & $12(66.7)$ & $50(92.6)$ & 127(84.7) & 0.063 \\
\hline Papillary & $1(1.7)$ & $2(11.1)$ & $2(11.1)$ & $1(1.9)$ & $6(4)$ & \\
\hline Medullary & $0(0)$ & $0(0)$ & $1(5.6)$ & $0(0)$ & $1(0.7)$ & \\
\hline metaplastic & $7(11.7)$ & $2(11.1)$ & $3(16.7)$ & $2(3.7)$ & $14(9.3)$ & \\
\hline Mixed & $1(1.7)$ & $0(0)$ & $0(0)$ & $1(1.9)$ & $2(1.3)$ & \\
\hline \multicolumn{7}{|l|}{ Tumor Grade } \\
\hline Grade-I & $1(1.7)$ & $0(0)$ & $0(0)$ & $0(0)$ & $1(0.7)$ & 0.118 \\
\hline Grade-II & $6(10)$ & $6(33.3)$ & $1(5.6)$ & $6(11.1)$ & 19(12.7) & \\
\hline Grade-III & 53(88.3) & $12(66.7)$ & 17(94.4) & 48(88.9) & 130(86.7) & \\
\hline \multicolumn{7}{|c|}{ Lymhovascular Invasion } \\
\hline Present & $13(21.7)$ & $6(33.3)$ & $7(38.9)$ & $10(18.5)$ & $36(24)$ & 0.250 \\
\hline Absent & $47(78.3)$ & $12(66.7)$ & $11(61.1)$ & $44(81.5)$ & $114(76)$ & \\
\hline \multicolumn{7}{|c|}{ Perinodal extension } \\
\hline Present & $12(20)$ & $2(11.1)$ & $6(33.3)$ & $10(18.5)$ & $30(20)$ & 0.436 \\
\hline Absent & $48(80)$ & 16(88.9) & $12(66.7)$ & $44(81.5)$ & $120(80)$ & \\
\hline \multicolumn{7}{|c|}{ Triple Negative phenotype } \\
\hline Basal & $6(10)$ & $2(11.1)$ & $2(11.1)$ & $6(11.1)$ & 16(10.7) & 1.000 \\
\hline Non Basal & $54(90)$ & 16(88.9) & 16(88.9) & 48(88.9) & 134(89.3) & \\
\hline
\end{tabular}

Chi-Square test applied

$P$-value $\leq 0.05$ considered as significant 
Table 3 Association of intensity of p53 overexpression with various clinical \& pathological parameters

\begin{tabular}{|c|c|c|c|c|c|c|}
\hline \multirow{2}{*}{+2} & \multicolumn{5}{|l|}{$\mathrm{n}(\%)$} & \multirow{2}{*}{$\begin{array}{l}P \text { - } \\
\text { Value }\end{array}$} \\
\hline & Weak $(n=21)$ & Intermediate $(n=26)$ & Strong $(n=57)$ & Negative $(n=46)$ & Total $(n=150)$ & \\
\hline \multicolumn{7}{|l|}{ Age groups } \\
\hline$\leq 30$ years & $0(0)$ & $0(0)$ & $3(5.3)$ & $2(4.3)$ & $5(3.3)$ & \multirow[t]{3}{*}{0.347} \\
\hline $31-50$ years & $8(38.1)$ & $14(53.8)$ & $34(59.6)$ & $28(60.9)$ & $84(56)$ & \\
\hline$>50$ years & $13(61.9)$ & $12(46.2)$ & $20(35.1)$ & $16(34.8)$ & $61(40.7)$ & \\
\hline \multicolumn{7}{|c|}{ Tumor stage/tumor size } \\
\hline $\mathrm{T} 1(\leq 2 \mathrm{~cm})$ & $6(28.6)$ & $5(19.2)$ & $14(24.6)$ & $1(2.2)$ & $26(17.3)$ & \multirow[t]{3}{*}{0.023} \\
\hline $\mathrm{T} 2(2.1-5.0 \mathrm{~cm})$ & $9(42.9)$ & $12(46.2)$ & $29(50.9)$ & $29(63)$ & $79(52.7)$ & \\
\hline $\mathrm{T} 3(>5.0 \mathrm{~cm})$ & $6(28.6)$ & $9(34.6)$ & $14(24.6)$ & $16(34.8)$ & $45(30)$ & \\
\hline \multicolumn{7}{|l|}{ ki67 index groups } \\
\hline$\leq 15 \%$ & $5(23.8)$ & $8(30.8)$ & $1(1.8)$ & $3(6.5)$ & $17(11.3)$ & \multirow[t]{4}{*}{0.006} \\
\hline $16-24 \%$ & $1(4.8)$ & $2(7.7)$ & $3(5.3)$ & $2(4.3)$ & $8(5.3)$ & \\
\hline $25-44 \%$ & $7(33.3)$ & $7(26.9)$ & $16(28.1)$ & 15 (32.6) & $45(30)$ & \\
\hline$>44 \%$ & $8(38.1)$ & $9(34.6)$ & $37(64.9)$ & $26(56.5)$ & $80(53.3)$ & \\
\hline \multicolumn{7}{|l|}{ Nodal Status } \\
\hline Positive & $9(42.9)$ & $13(50)$ & $21(36.8)$ & $21(45.7)$ & $64(42.7)$ & \multirow[t]{2}{*}{0.675} \\
\hline Negative & $12(57.1)$ & $13(50)$ & $36(63.2)$ & $25(54.3)$ & $86(57.3)$ & \\
\hline \multicolumn{7}{|l|}{ Nodal Stage } \\
\hline No & $12(57.1)$ & $13(50)$ & $36(63.2)$ & $27(58.7)$ & $88(58.7)$ & \multirow[t]{4}{*}{0.357} \\
\hline $\mathrm{N} 1$ & $5(23.8)$ & $7(26.9)$ & $8(14)$ & $10(21.7)$ & $30(20)$ & \\
\hline N2 & $0(0)$ & $5(19.2)$ & $5(8.8)$ & $3(6.5)$ & $13(8.7)$ & \\
\hline N3 & $4(19)$ & $1(3.8)$ & $8(14)$ & $6(13)$ & 19 (12.7) & \\
\hline \multicolumn{7}{|c|}{ Histological Subtypes } \\
\hline IDC & $17(81)$ & $21(80.8)$ & $50(87.7)$ & $39(84.8)$ & $127(84.7)$ & \multirow[t]{5}{*}{0.620} \\
\hline Papillary & $1(4.8)$ & $1(3.8)$ & $3(5.3)$ & $1(2.2)$ & $6(4)$ & \\
\hline Medullary & $0(0)$ & $1(3.8)$ & $0(0)$ & $0(0)$ & $1(0.7)$ & \\
\hline metaplastic & $2(9.5)$ & $3(11.5)$ & $3(5.3)$ & $6(13)$ & $14(9.3)$ & \\
\hline Mixed & $1(4.8)$ & $0(0)$ & $1(1.8)$ & $0(0)$ & $2(1.3)$ & \\
\hline \multicolumn{7}{|l|}{ Tumor Grade } \\
\hline Grade-I & $1(4.8)$ & $0(0)$ & $0(0)$ & $0(0)$ & $1(0.7)$ & \multirow[t]{3}{*}{0.041} \\
\hline Grade-II & $6(28.6)$ & $4(15.4)$ & $6(10.5)$ & $3(6.5)$ & $19(12.7)$ & \\
\hline Grade-III & $14(66.7)$ & $22(84.6)$ & $51(89.6)$ & $43(93.5)$ & $130(86.7)$ & \\
\hline \multicolumn{7}{|c|}{ Lymhovascular Invasion } \\
\hline Present & $7(33.3)$ & $6(23.1)$ & $15(26.3)$ & $8(17.4)$ & $36(24)$ & \multirow[t]{2}{*}{0.516} \\
\hline Absent & $14(66.7)$ & $20(76.9)$ & $42(73.7)$ & $38(82.6)$ & $114(76)$ & \\
\hline \multicolumn{7}{|l|}{ Perinodal extension } \\
\hline Present & $5(23.8)$ & $3(11.5)$ & $15(26.3)$ & $7(15.2)$ & $30(20)$ & \multirow[t]{2}{*}{0.352} \\
\hline Absent & $16(76.2)$ & $23(88.5)$ & $42(73.7)$ & $39(84.8)$ & $120(80)$ & \\
\hline \multicolumn{7}{|c|}{ Triple Negative phenotype } \\
\hline Basal & $3(14.3)$ & $3(11.5)$ & $5(8.8)$ & $5(10.9)$ & $16(10.7)$ & \multirow[t]{2}{*}{0.913} \\
\hline Non Basal & 18 (85.7) & $23(88.5)$ & $52(91.2)$ & 41 (89.1) & 89.3) & \\
\hline
\end{tabular}

Chi-Square test applied

$P$-value $\leq 0.05$ considered as significant 
association of either p16 or p53 over-expression was noted with recurrence status of patients (Fig. 2).

\section{Discussion}

In the present study, high expression of p16 was noted in TNBC cases while a moderately high expression of p53 was also notable. Moreover, p53 over-expression significantly correlated with key prognostic factors of breast cancer like T-stage, $\mathrm{N}$-stage, tumor grade and ki67 index.

Breast cancers are quite frequent in Southeast Asia and typically associated with adverse prognostic features [17-20]. Multiple studies investigated the prognostic significance of p53 mutations in breast cancer. Somatic mutations of p53 (TP53) are found in $20-30 \%$ of breast cancer [21], while germ-line mutations are relatively rare. Although, the predictive value of TP53 abnormalities is still unclear, somatic TP53 mutations signify worse prognosis independent of tumor size and nodal status [22]. A study involving 1800 patients of breast cancer revealed twice higher risk of death in tumors having TP53 mutations [23]. A similar association of p53 IHC expression with bad prognosis in breast cancer is debatable as cutoff values have not been defined and ASCO panel still don't advice routine p53 IHC expression testing in breast cancer. However, as mutated p53 protein is not digested quickly inside tumor cells as compared to wild type protein, and therefore accumulates inside tumor cells. Hence, it is reasonable to consider high p53 expression as a surrogate marker of TP53 mutation. Moreover, as various biomarker testing have now been shifted to IHC, therefore with the help of results of various ongoing research, p53 IHC may get incorporated in future ASCO/CAP recommendations. Furthermore, gene expression analysis studies revealed that p53 and other tumor suppressor DNA repair gene mutation and aberrant expression in TNBC may have important clinical implications as they may effect sensitivity to platinum \& other chemotherapeutic agents that are directly DNA damaging [24, 25].

Unlike p53, prognostic significance of p16 in TNBC is more controversial; however, high expression of p16 has been noted in various studies [26]. A study involving 60 TNBC cases revealed high ki67 index in p16 positive tumors regardless of p53 expression. As high ki67 index is a well defined prognostic factor in breast cancer [27], therefore they suggested a potential prognostic value of p16 over-expression in TNBC [28]; however, we didn't find any such association. Basal type phenotype of TNBC is a worse subtype of breast cancer with high expression of CK5/6 (Table 4). Frequency of basal subtype of TNBC in different areas of world is different; we found a low proportion of basal subtype in our study (10\%). A study involving $85 \%$ of TNBC revealed a high expression of p16 in basal subtype as compared to non-basal phenotype ( $80 \%$ vs. $50.8 \%$ respectively) [29]; however, no such association was noted in our study.

One of the limitations of our study was that molecular testing of p16 \& p53 was not performed, therefore we suggest molecular testing of p16 \& p53 in TNBC of our population to establish mutation status and its correlation with IHC over-expression of these biomarkers. Moreover, we didn't find any significant correlation of recurrence status of TNBC with p53 \&p16 over-expression; however it can't be concluded that there is no correlation of p53 expression with recurrence status, as other important factors determining recurrence like margin status of tumors was not taken into account.

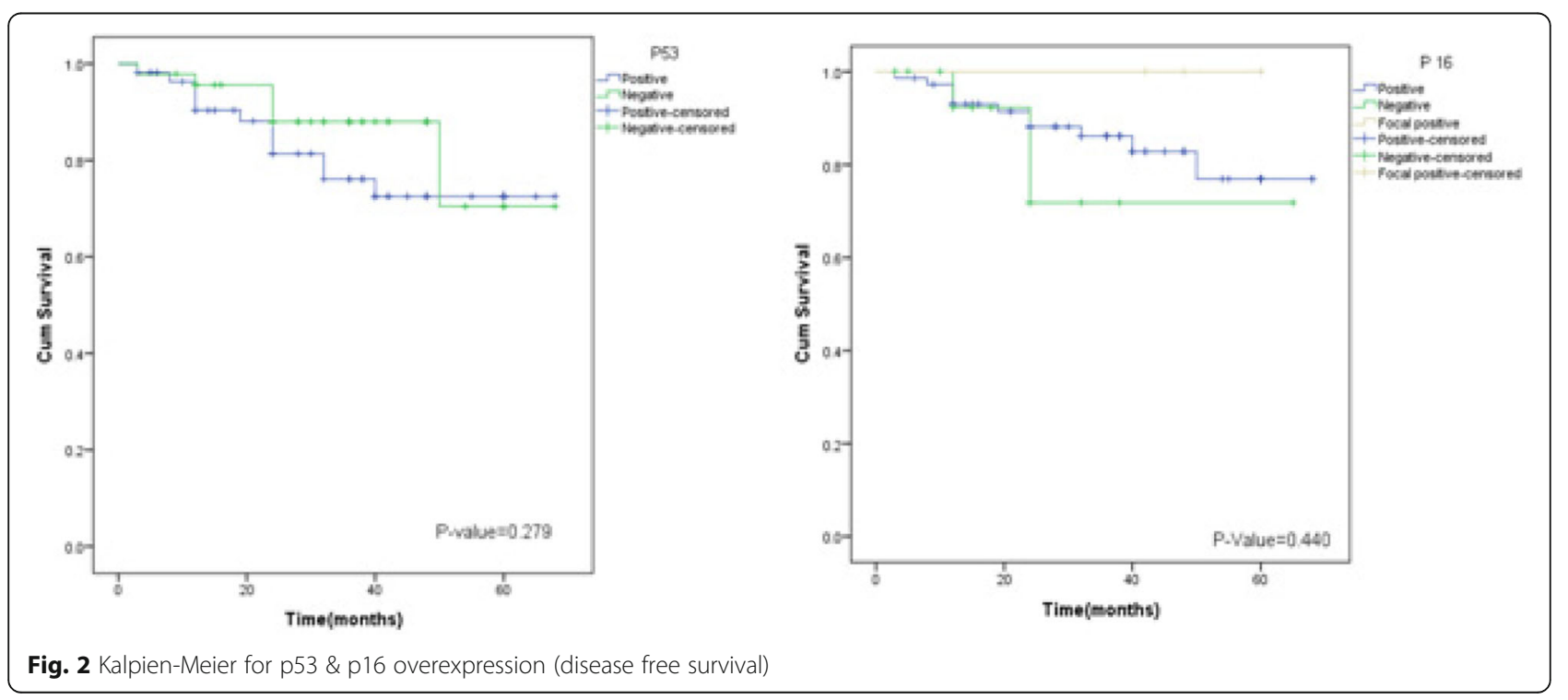


Table 4 Association of p16 overexpression with various clinical \& pathological parameters

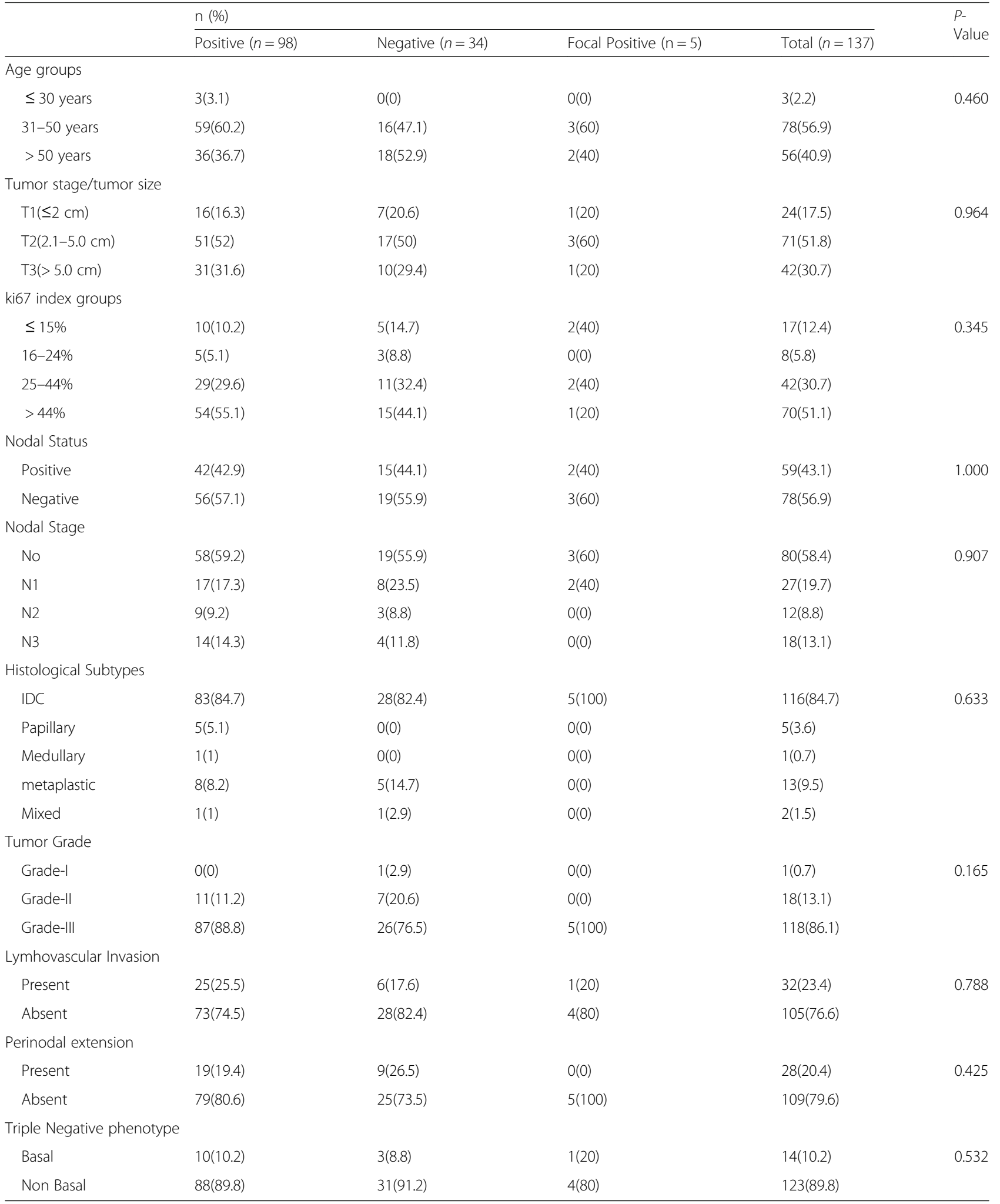

Chi-Square test applied

$P$-Value $\leq 0.05$, considerd as significant 


\section{Conclusion}

On the basis of significant association of p53 IHC over-expression with worse prognostic factors in TNBC, therefore we suggest that more large scale studies are needed to validate this finding in loco-regional population. Moreover, high expression of p16 in TNBC suggests a potential role of this biomarker in TNBC pathogenesis which should be investigated with molecular based research in our population.

\section{Acknowledgments}

We gratefully acknowledge all staff members of Pathology, Liaquat National Hospital, Karachi, Pakistan for their help and cooperation.

\section{Availability of data and materials}

Please contact author, Atif Ali Hashmi (doc_atif2005@yahoo.com) for data requests.

\section{Authors' contributions}

$\mathrm{AAH}$ and SN: main author of manuscript, have made substantial contributions to conception and design of study. SKH, ZFH and MI: have been involved in requisition of data. EYK, NF, AK and MME have been involved in analysis of the data and revision of the manuscript. All authors read, revise and gave approval of the manuscript

\section{Ethics approval and consent to participate}

Ethics committee of Liaquat National Hospital, Karachi, Pakistan approved the study. Written informed consent was obtained from the patients for the participation

\section{Consent for publication}

Not applicable.

\section{Competing interests}

The authors declare that they have no competing interests.

\section{Publisher's Note}

Springer Nature remains neutral with regard to jurisdictional claims in published maps and institutional affiliations.

\section{Author details}

'Liaquat National Hospital and Medical College, Karachi, Pakistan. ${ }^{2} \mathrm{CMH}$ Institute of Medical Sciences, Multan, Pakistan. ${ }^{3}$ Kandahar University, North, Kandahar 3802, Afghanistan. ${ }^{4}$ Brown University, Providence, RI, USA.

Received: 26 January 2018 Accepted: 26 September 2018

Published online: 03 October 2018

\section{References}

1. Swain S. Triple-negative breast Cancer: metastatic risk and role of platinum agents 2008 ASCO clinical science symposium, 2008. June 3, 2008.

2. Hashmi AA, Edhi MM, Naqvi H, Khurshid A, Faridi N. Molecular subtypes of breast cancer in South Asian population by immunohistochemical profile and Her2neu gene amplification by FISH technique: association with other clinicopathologic parameters. Breast J. 2014:20(6):578-85.

3. Hammond ME, Hayes DF, Dowsett M, et al. American Society of Clinical Oncology/College of American Pathologists guideline recommendations for immunohistochemical testing of estrogen and progesterone receptors in breast cancer (unabridged version). Arch Pathol Lab Med. 2010;134:e48.

4. Hammond ME, Hayes DF, Dowsett M, et al. American Society of Clinical Oncology/college of American pathologists guideline recommendations for immunohistochemical testing of estrogen and progesterone receptors in breast cancer. J Clin Oncol. 2010;28:2784.

5. Wolff AC, Hammond ME, Hicks DG, et al. Recommendations for human epidermal growth factor receptor 2 testing in breast cancer: American Society of Clinical Oncology/College of American Pathologists clinical practice guideline update. J Clin Oncol. 2013;31:3997.
6. Hashmi AA, Edhi MM, Naqvi H, Faridi N, Khurshid A, Khan M. Clinicopathologic features of triple negative breast cancers: an experience from Pakistan. Diagn Pathol. 2014;9:43

7. Hashmi AA, Naz S, Hashmi SK, Hussain ZF, Irfan M, Bakar SMA, Faridi N, Khan A, Edhi MM. Cytokeratin 5/6 and cytokeratin 8/18 expression in triple negative breast cancers: clinicopathologic significance in South-Asian population. BMC Res Notes. 2018;11(1):372.

8. Lehmann BD, Bauer JA, Chen X, et al. Identification of human triplenegative breast cancer subtypes and preclinical models for selection of targeted therapies. J Clin Invest. 2011;121:2750.

9. Prat A, Parker JS, Karginova O, et al. Phenotypic and molecular characterization of the claudin-low intrinsic subtype of breast cancer. Breast Cancer Res. 2010:12:R68.

10. Livasy CA, Karaca G, Nanda R, et al. Phenotypic evaluation of the basal-like subtype of invasive breast carcinoma. Mod Pathol. 2006;19:264

11. Nielsen TO, Hsu FD, Jensen $K$, et al. Immunohistochemical and clinical characterization of the basal-like subtype of invasive breast carcinoma. Clin Cancer Res. 2004;10:5367.

12. Montanari M, Boninsegna A, Faraglia B, Coco C, Giordano A, et al. Increased expression of geminin stimulates the growth of mammary epithelial cells and is a frequent event in human tumors. J Cell Physiol. 2005;202:215-22

13. Zhang J, Pickering CR, Holst CR, Gauthier ML, Tlsty TD. p16INK4a modulates p53 in primary human mammary epithelial cells. Cancer Res. 2006;66:10325-31.

14. Hashmi AA, Hussain ZF, Hashmi SK, Irfan M, Khan EY, Faridi N, Khan A, Edhi MM. Immunohistochemical over expression of p53 in head and neck Squamous cell carcinoma: clinical and prognostic significance. BMC Res Notes. 2018;11(1):433.

15. Bartley AN, Ross DW. Validation of p53 immunohistochemistry as a prognostic factor in breast cancer in clinical practice. Arch Pathol Lab Med. 2002:126:456-8

16. Hui R, Macmillan RD, Kenny FS, Musgrove EA, Blamey RW, et al. INK4a gene expression and methylation in primary breast cancer: overexpression of p16INK4a messenger RNA is a marker of poor prognosis. Clin Cancer Res. 2000;6:2777-87.

17. Hashmi AA, Mahboob R, Khan SM, Irfan M, Nisar M, Iftikhar N, Siddiqui M, Faridi N, Khan A, Edhi MM. Clinical and prognostic profile of Her2neu positive (non-luminal) intrinsic breast cancer subtype: comparison with Her2neu positive luminal breast cancers. BMC Res Notes. 2018;11(1):574

18. Hashmi AA, Aijaz S, Mahboob R, Khan SM, Irfan M, Iftikhar N, Nisar M, Siddiqui M, Edhi MM, Faridi N, Khan A. Clinicopathologic features of invasive metaplastic and micropapillary breast carcinoma: comparison with invasive ductal carcinoma of breast. BMC Res Notes. 2018:11(1):531.

19. Hashmi AA, Aijaz S, Khan SM, Mahboob R, Irfan M, Zafar NI, Nisar M, Siddiqui M, Edhi MM, Faridi N, Khan A. Prognostic parameters of luminal A and luminal B intrinsic breast cancer subtypes of Pakistani patients. World J Surg Oncol. 2018;16(1):1.

20. Hashmi AA, Faridi N, Khurshid A, Naqvi H, Malik B, Malik FR, Fida Z, Mujtuba S. Accuracy of frozen section analysis of sentinel lymph nodes for the detection of Asian breast cancer micrometastasis - experience from Pakistan. Asian Pac J Cancer Prev. 2013:14(4):2657-62.

21. Petitjean A, Achatz MI, Borresen-Dale AL, et al. TP53 mutations in human cancers: functional selection and impact on cancer prognosis and outcomes. Oncogene. 2007;26:2157.

22. Bonnefoi H, Piccart M, Bogaerts J, et al. TP53 status for prediction of sensitivity to taxane versus non-taxane neoadjuvant chemotherapy in breast cancer (EORTC 10994/BIG 1-00): a randomised phase 3 trial. ancet Oncol. 2011:12:527.

23. Olivier $M$, Langerød $A$, Carrieri $P$, et al. The clinical value of somatic TP53 gene mutations in 1,794 patients with breast cancer. Clin Cancer Res. 2006;12:1157

24. Sørlie T, Perou CM, Tibshirani R, et al. Gene expression patterns of breast carcinomas distinguish tumor subclasses with clinical implications. Proc Nat Acad Sci U S A. 2001:98:10869.

25. Troester MA, Herschkowitz J, Oh DS, et al. Gene expression pattern associated with p53 status in breast cancer. BMC Cancer. 2006:6:276.

26. Shan M, Zhang X, Liu X, Qin Y, Liu T, Liu Y, Wang J, Zhong Z, Zhang Y, Geng J, Pang D. P16 and p53 play distinct roles in different subtypes of breast cancer. PLoS One. 2013;8(10):e76408. 
27. Haroon S, Hashmi AA, Khurshid A, Kanpurwala MA, Mujtuba S, Malik B Faridi N. Ki67 index in breast cancer: correlation with other prognostic markers and potential in pakistani patients. Asian Pac J Cancer Prev. 2013;14(7):4353-8.

28. Sugianto J, Sarode V, Peng Y. Ki-67 expression is increased in p16-expressing triple-negative breast carcinoma and correlates with p16 only in p53-negative tumors. Hum Pathol. 2014;45(4):802-9.

29. Abou-Bakr AA, Eldweny HI. p16 expression correlates with basal-like triplenegative breast carcinoma. Ecancermedicalscience. 2013;7:317.

Ready to submit your research? Choose BMC and benefit from:

- fast, convenient online submission

- thorough peer review by experienced researchers in your field

- rapid publication on acceptance

- support for research data, including large and complex data types

- gold Open Access which fosters wider collaboration and increased citations

- maximum visibility for your research: over $100 \mathrm{M}$ website views per year

At $\mathrm{BMC}$, research is always in progress.

Learn more biomedcentral.com/submissions 